\author{
РАЗРАБОТКА МОДЕЛИ БИОДЕГРАДАЦИИ \\ МНОГОКОМПОНЕНТНЫХ УГЛЕВОДОРОДНЫХ НЕФТЯНЫХ \\ СОЕДИНЕНИЙ
}

\title{
BIODEGRADATION OF MULTICOMPONENT HYDROCARBON PETROLEUM COMPOUNDS MODEL DEVELOPMENT
}

\author{
Р.Ш. Маммадли \\ Национальное аэрокосмическое агентство, \\ г. Баку, Азербайджанская Республика
}

Rashad Sh. Mammadli

National Aerospace Agency, Baku, Azerbaijan Republic

Аннотация. Рассмотрены вопросы разработки модели биодеградации многокомпонентных углеводородных соединений. Поставлена и решена задача математического описания кинетики деградирующих компонентов нефти. Учитывая то, что в смеси углеводородов деградация одной компоненты может ингибировать другую компоненту, построена оптимизационная математическая модель, отображающая кинетику процесса. Найдено условие, при котором среднеинтегрированная величина остаточных концентраций в период роста среднегеометрической величины исходных концентраций достигает минимума.

Abstract. The issues of developing a model of biodegradation of multicomponent hydrocarbon compounds are considered. The problem of mathematical description of the kinetics of degrading oil components has been set and solved. Due to the fact that degradation of one component in a mixture of hydrocarbons can inhibit another component, an optimization mathematical model reflecting the kinetics of the process has been built. A condition under 
which the average integrated value of residual concentrations reaches a minimum during the period of growth of the geometric average value of the initial concentrations has been found.

Ключевые слова: кинетика; биодеградация; углеводороды; концентрация; модель

Key words: kinetics; biodegradation; hydrocarbons; concentration; model

\section{Введение}

Хорошо известно [1], что простая модель кинетики первого порядка описывается следующим уравнением:

$$
\ln \left(\frac{C}{C_{0}}\right)=k \cdot t_{k},
$$

где $C$ - текущая концентрация вещества, подвергнувшегося деградации (мг/кг);

$t_{k}$ - время деградации (ч);

$C_{0}$ - исходная концентрация вещества (мг/кг);

$k$ - постоянная изменения концентрация вещества в какой-либо среде $\left(\mathrm{ч}^{-1}\right)$.

Как отмечается в работе [2], причины наличия различий в скорости деградации различных соединений имеют комплексный характер и включают такие факторы, как термодинамические показатели, способность химической структуры противостоять усилиям энзимов разрушить углеводородные связи; насколько легко различные составляющие транспортируются в ячейку и т.д. В качестве примера на рисунке 1 приведены кривые деградации различных углеводородных соединений. 


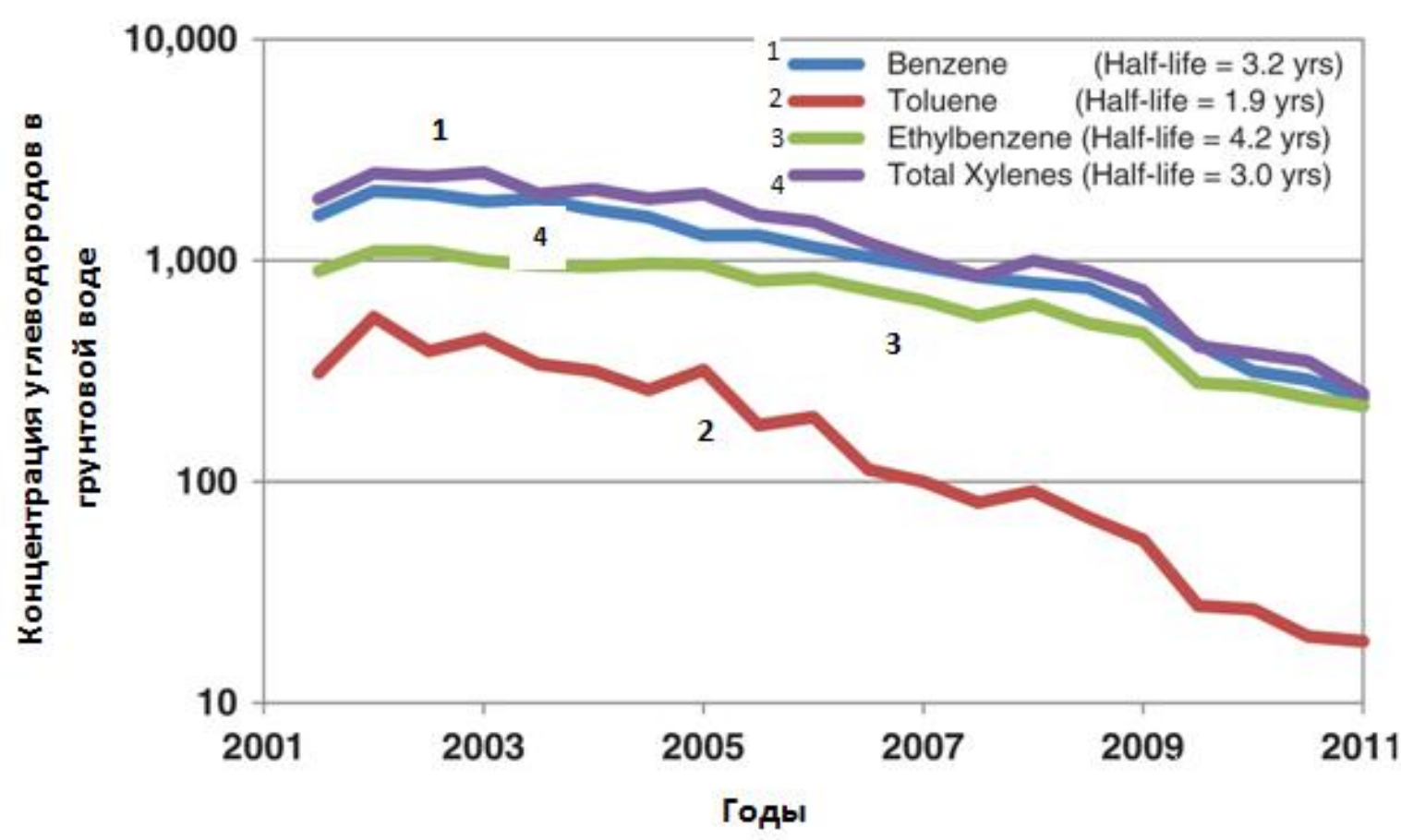

1 - бензин, время деградации 3,2 года; 2 - толуол, время деградации 1,9 года; 3 - этилбензал, время деградации 4,2 года; 4 - ксилены, время деградации 3,0 года

Рисунок 1. Изменение концентрации углеводородов группы ВТЕХ во времени в грунтовых водах. Измерения проводились в зоне заправочной станции в Калифорнии с 2001 по 2011 гг.

Как отмечается в [1], биодеградация нефтяных углеводородов является природным процессом, включающим протекание каталитических реакций между микробиальными и химическими материалами, имеющимися в рассматриваемой природной среде. При этом, согласно [2], скорость деградации нефтяных углеводородов управляется одним или несколькими факторами (например температура, $p H$ и др.). Согласно [3], некоторые углеводороды не подвержены биодеградации. Как отмечается в [4], процесс биодеградации нефтяных углеводородов может быть смоделирован процессом кинетики первого порядка. При этом, согласно [5], некоторые углеводородные составляющие могут ингибировать биодеградацию других углеводородов, что также подтверждается экспериментальными результатами, приведенными в [1].

Целью настоящего исследования является формирование оптимизационной кинетической модели совместной биодеградации 
углеводородных компонентов нефти, в которой оптимальная кинетическая взаимосвязь компонентов обеспечила бы достижение минимальной величины оставшегося количества углеводородов, не подвергнувшихся деградации.

\section{Материалы и методы}

Уравнение (1) легко можно преобразовать в следующий вид:

$$
C=C_{0} \cdot e^{-k k_{k}}
$$

Если рассматриваются несколько углеводородных компонентов, то имеем:

$$
\begin{aligned}
& C_{1}=C_{01} \cdot e^{-k_{1} t_{k}} \\
& C_{2}=C_{02} \cdot e^{-k_{2} t_{k}} \\
& C_{n}=C_{0 n} \cdot e^{-k_{n} t_{k}}
\end{aligned}
$$

Для дальнейшего анализа воспользуемся методом двойного усреднения, предложенного авторами статьи [6]. Согласно этому методу должны быть осуществлены следующие операции:

1. Вычисление взвешенной геометрического среднего $A$ показателей $C_{i}, i=(\overline{i, n})$.

2. Выбор искомой функции - скорости деградации компонент, которая способна ингибировать биодеградацию одних компонентов и в то же время стимулировать рост скорости биодеградации других компонентов. Графически это условие иллюстрировано на рисунке 2. 


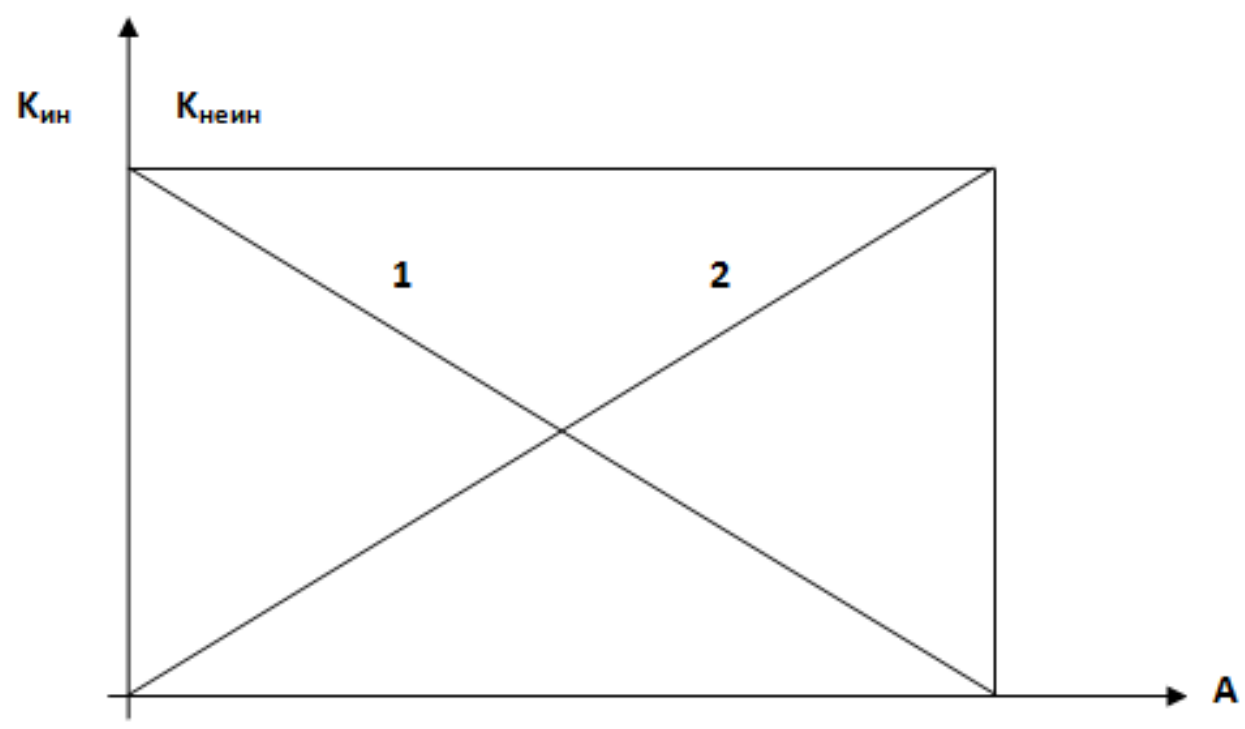

1 - скорость ингибируемых компонентов;

2 - скорость не ингибированных компонентов

Рисунок 2. Возможная взаимосвязь скоростей биодеградации углеводородных компонентов нефти

Чисто физически указанное условие означает, что если под воздействием некоторых внешних факторов $\left(t^{0}, p H\right.$, и т.д.) $K_{i}$ растет, то в результате ингибирования скорость биодеградации $j$-й компоненты уменьшается. Сформулируем ограничительное условие, содержащее функции:

$$
\begin{aligned}
& K_{1}=\varphi_{1}(A) ; \\
& K_{2}=\varphi_{2}(A),
\end{aligned}
$$

где (4) указывает зависимость скорости деградации ингибируемой компоненты при росте $A$ - взвешенной геометрически усредненной величины концентраций всех компонентов; (5) указывает зависимость скорости деградации ингибирующей компоненты при росте $A$.

Сформулированное ограничительное условие имеет вид:

$$
F_{1}=\int_{0}^{A_{\max }}\left[d_{1} \varphi_{1}(A)+d_{2} \varphi_{2}(A)\right] d A=C ; \quad C=\text { const },
$$

где $d_{1}$ и $d_{2}$ - экспериментально определяемые коэффициенты. 
3. Составление основного функционала оптимизации в виде

$$
F_{1}=\int_{0}^{A_{\max }} A \exp \left[-\left(m_{1} k_{1} t_{1}+m_{2} k_{2} t_{2}\right)\right] d A
$$

где $m_{1}, m_{2}-$ весовые коэффициенты; определяемые опытным путем.

$$
A=C_{01}^{m_{1}} \cdot C_{02}^{m_{2}} .
$$

Полный функционал $F_{2}$ безусловной вариационной оптимизации с учетом (6) и (7) имеет вид:

$$
F_{2}=F_{1}+\lambda\left(F_{2}-C\right) .
$$

С учетом (6) и (7) имеем

$$
F_{2}=\int_{0}^{A_{\max }} A \exp \left[-\left(m_{1} \varphi_{1}(A) t+m_{2} \varphi_{2}(A) t\right)\right]+\lambda\left[d_{1} \varphi_{1}(A)+d_{2} \varphi_{2}(A)-C\right],
$$

где $\lambda$ - множитель Лагранжа.

Считая $\varphi_{2}(A)$ известной функцией, вычислим оптимальную взаимосвязь функций $\varphi_{1}(A)$ и $\varphi_{2}(A)$, при которой $F_{2}$ достиг бы экстремального значения.

\section{Решение сформированной оптимизационной задачи}

Согласно [7], решение сформированной оптимизационной задачи должно удовлетворять условию:

$$
\frac{d\left\{A \cdot \exp \left[-\left(m_{1} \phi_{1}(A) t+m_{2} \phi_{2}(A) t\right]+\lambda\left[d_{1} \phi_{1}(A)+d_{2} \phi_{2}(A)-C\right]\right\}\right.}{d \phi_{1}(A)}=0 .
$$

Из (11) находим

$$
-A \cdot \exp \left[-\left(m_{1} \varphi_{1}(A) t+m_{2} \varphi_{2}(A) t\right] m_{1} t+\lambda \cdot d_{1}=0 .\right.
$$

Из (12) находим

$$
\exp \left[-\left(m_{1} \phi_{1}(A) t+m_{2} \phi_{2}(A) t\right]=\frac{\lambda d_{1}}{A \cdot m_{1} \cdot t} .\right.
$$


Из (13) получим

$$
\left(m_{1} \phi_{1}(A) t+m_{2} \phi_{2}(A) t\right)=\ln \frac{A m_{1} t}{\lambda d_{1}} .
$$

Из (14) находим

$$
\phi_{1}(A)=\frac{\ln \frac{A \cdot m_{1} t}{\lambda d_{1}}}{m_{1} t}-\frac{m_{2} \phi_{2}(A)}{m_{1}} .
$$

Для вычисления конкретного значения $\lambda$ достаточно решение (15) вставить в формулу (6), осуществить интегрирование и вычислить значение $\lambda$.

Не вдаваясь в математические подробности процесса вычисления этого множителя, для проверки типа экстремума функционала (10) достигаемого при решении (15) вычислим знак второй производной интегранта в (10) по $\varphi_{1}(A)$. Вычисление показывает, что указанная вторая производная имеет плюсовой знак, т.е. концентрация исследуемой смеси углеводородов при решении (15) достигает минимума.

\section{Вывод}

Таким образом, сформулирована и решена оптимизационная задача моделирования деградирующих компонентов нефти. На основе известного факта о том, что в смеси углеводородов деградация одной компоненты может ингибировать другую компоненту, построена оптимизационная математическая модель, отображающая кинетику процесса. Вычислено условие, при которой среднеинтегрированная величина остаточных концентраций в период роста среднегеометрической величины исходных концентраций достигает минимума. 


\section{Список используемых источников}

1. Yang M., Yang Y.S., Du X., Cao Y., Lei Y. Fate and Transport of Petroleum Hydrocarbons in Vadose Zone: Compound-Specific Natural Attenuation // Water, Air and Soil Pollution. 2013. Vol. 224. P. 1439. DOI 10.1007/s11270-013-1439-y.

2. Garg S., Newell C.J., Kulkarni P.R., King D.C., Adamson D.T., Renno M.I., Sale T. Overview of Natural Source Zone Depletion: Processes, Controlling Factors and Composition Change // Ground Monitoring and Remediation. 2017. Vol. 37. Issue 3. P. 62-81. DOI: 10.1111/gwmr.12219.

3. Salanitro J.P. Bioremediation of Petroleum Hydrocarbons in Soil // Advanced in Agronomy. 2001. Vol. 72. P. 53-105. DOI: 10.1016/S00652113(01)72011-1.

4. Suarez M.P., Rifai H.S. Biodegradation Rates for Fuel Hydrocarbons and Chlorinated Solvents in Groundwater // Bioremediation Journal. 1999. Vol. 3. Issue 4. P. 337-362. DOI: 10.1080/10889869991219433.

5. Sherry A., Grant R., Aitken C., Jones M., Head I., Gray N. Volatile Hydrocarbons Inhibit Methanogenic Crude Oil Degradation // Frontiers in Microbiology. 2014. Vol. 5. P. 131. DOI: 10.3389/fmicb.2014.00131.

6. Джавадов Н.Г., Асадов Х.Г., Казымлы Р.В. Метод двойного усреднения для минимизации неопределённости результатов измерений парниковых газов в наземных распределённых сетях атмосферных измерений // Метрология. 2020.№2. С. 19-30. DOI: 10.32446/01324713.2020-2-19-30.

7. Эльсгольц Л.Э. Дифференциальные уравнения и вариационное исчисление. М.: Наука, 1974. 432 с. 


\section{References}

1. Yang M., Yang Y.S., Du X., Cao Y., Lei Y. Fate and Transport of Petroleum Hydrocarbons in Vadose Zone: Compound-Specific Natural Attenuation. Water, Air and Soil Pollution, 2013, Vol. 224, pp. 1439. DOI 10.1007/s11270-013-1439-y.

2. Garg S., Newell C.J., Kulkarni P.R., King D.C., Adamson D.T., Renno M.I., Sale T. Overview of Natural Source Zone Depletion: Processes, Controlling Factors and Composition Change. Ground Monitoring and Remediation, 2017, Vol. 37, Issue 3, pp. 62-81. DOI: 10.1111/gwmr.12219.

3. Salanitro J.P. Bioremediation of Petroleum Hydrocarbons in Soil. Advanced in Agronomy, 2001, Vol. 72, pp. 53-105. DOI: 10.1016/S00652113(01)72011-1.

4. Suarez M.P., Rifai H.S. Biodegradation Rates for Fuel Hydrocarbons and Chlorinated Solvents in Groundwater. Bioremediation Journal, 1999, Vol. 3, Issue 4, pp. 337-362. DOI: 10.1080/10889869991219433.

5. Sherry A., Grant R., Aitken C., Jones M., Head I., Gray N. Volatile Hydrocarbons Inhibit Methanogenic Crude Oil Degradation. Frontiers in Microbiology, 2014, Vol. 5, pp. 131. DOI: 10.3389/fmicb.2014.00131.

6. Dzhavadov N.G., Asadov Kh.G., Kazymly R.V. Metod dvoinogo usredneniya dlya minimizatsii neopredelennosti rezul'tatov izmerenii parnikovykh gazov $\mathrm{v}$ nazemnykh raspredelennykh setyakh atmosfernykh izmerenii [Method of Double Averaging for Optimum Accounting of NonCertainty of Results of Measurements Greenhouse Gases Low Gases Concentrations at the Ground Distributed Systems of Atmospheric Measurements]. Metrologiya-Measurement Techniques, 2020, No. 2, pp. 19-30. DOI: 10.32446/0132-4713.2020-2-19-30. [in Russian].

7. Elsgolts L.E. Differentsial'nye uravneniya $i$ variatsionnoe ischislenie [Differential Equations and Calculus of Variations]. Moscow, Nauka Publ., 1974. 432 p. [in Russian]. 


\section{Сведения об авторе}

\section{About the author}

Маммадли Рашад Шохрат оглу, аспирант Национального аэрокосмического агентства, г. Баку, Азербайджанская Республика

Rashad Sh. Mammadli, Post-Graduate Student of National Aerospace Agency, Baku, Azerbaijan Republic 